\title{
Superiority of Concurrent Chemo Radiation Over Sequential Chemo Radiotherapy for Palliation of Dysphagia in Carcinoma Esophagus, A Reterospective Study.
}

\author{
Dr Pramila Kumari ${ }^{1}$,Dr Praveen Kumar Khatri ${ }^{1}$, ,Dr Saroj Kumari ${ }^{1}$, Dr Mukesh \\ Kumar Singhal ${ }^{1}$,Dr Kamlesh Kumar Harsh ${ }^{3}$,Dr Shankar Lal Jakhar ${ }^{2}$, \\ Dr Neeti Sharma ${ }^{2}$,Dr HS Kumar ${ }^{4}$ \\ Medical Officer ${ }^{1}$, Associate Professor ${ }^{2}$, Assistant Professor ${ }^{3}$, Head Of Department And Senior Professor ${ }^{4}$ \\ Department Of Radiation Oncology, SP Medical College And Associated Group Of Hospital, Bikaner, \\ Rajasthan, India
}

\begin{abstract}
Aim: Positive results of many Trials have incorporated concurrent and sequential chemo radiotherapy in treatment of inoperable carcinoma esophagus. But concurrent chemo radiation therapy is similar to that achieved by surgery alone. The main plea of concurrent chemoradiation in esophageal cancers is early regression and palliation of dysphagia for long time.

The aim of this study to analyse the modalities of treatment available for palliation of dysphagia in carcinoma esophagus and determine the most effective option among them.

Materials and Methods: Between September 2011 to December 2013,50 patients of esophageal cancers were treated in our institute, 25 patients in sequential and 25 in concurrent chemo radiotherapy arm. Swallowing function was assessed in these patients by the use of a swallowing-function scoring system.

Results: Assessment of response in two arms were done for grade of dysphagia palliation, Complete Response at 6 months and toxicity. Dysphagia scores improved in $88 \%$ in study arm and $64 \%$ in control arm. In study arm $28 \%$ male and $52 \%$ female patients, while in control arm $40 \%$ male and $28 \%$ female patients presented with $C R$ in primary tumor and mediastinal lymph node. There was statistical significant difference in toxicities of TLC and ANC between both arms. Grade 2 and 3 toxicities were $40 \%$ and $4 \%$ for TLC and were $28 \%$ and $0 \%$ for ANC in study and control arm respectively $(p=0.013, p=.014)$. This may be due to concurrent use of chemotherapy with radiation in study arm that also showed synergism for toxicity of TLC and ANC.

Conclusion: Concurrent Chemo Radiotherapy is a more aggressive approach for dysphagia control, which is beneficial for those patients with good performance status.This approach is used as an alternative to stenting. This combination is more effective than neoadjuvant chemoradiation for improving dysphagia scores and QoL in inoperable esophageal cancers .
\end{abstract}

Keywords: Chemo radiation, comparison, esophagus, sequential and concurren

\section{Introduction}

Esophageal cancer is the sixth leading cause of cancer deaths in the world, and major burden of disease present in developing countries. ${ }^{[1]}$ Worldwide, Squamous cell carcinoma histology present in $90 \%$ of esophageal cancers, approximately 5\% are Adenocarcinoma, the remaining 5\% represent metastases from other organs and rare malignancies. Factors which causing esophageal cancer are multitudinous and mingled. Familial aggregation for esophageal cancers is not clear and the main reasons appears to be combinations of lifestyle factors, dietery and enviromental. [2] Predominant histology in the cervical esophagus, upper and middle thirds of the thoracic esophagus is Squamous cell carcinoma, whereas adenocarcinoma in the distal esophagus [3].Approximately $90 \%$ of patients from dysphagia and 50\% patients suffer from Odynophagia (pain on swallowing) regardless of location and histology. Next common feature is Weight loss, if this loss is $>5 \%$ of total body weight, associated with a worse prognosis. Less frequent symptoms may include anorexia, cough, hoarseness. [4] As the disease advances in these patients, leads to reduced lumen of esophagus subsequently development of dysphagia,pain ,poor neutrition ultimately lower score of performance and quality of life.[5]

Squamous cell carcinoma histology predominant in the upper and middle thirds of the esophagus, locoregional recurrence in these patients are mainly locoregional. Rich lymphatic chennels ( submucosal) and the lack of a serosal envelope of the esophagus provide a favorable way for development of locally advanced infiltration by tumor and extensive lymphadenopathy. [6] whereas distant recurrence are commonly seen in patients with lesions of the lower third, where predominant histology is adenocarcinomas, common sites for distant metastasis are lung, liver and bone. Less common territories are subcutaneous region and brain. [7] 
Locally advanced carcinoma esophagus has 5 year survival only $10 \%$ to $15 \%$ despite the use of combined modality either surgical, medical or radiation oncology. The majority of patients with these cancers will require some form of palliative treatment. [8-11] Updated trial results of Al-Sarraf et al. showed that result of combined chemo and radiotherapy is equivalent to surgery alone. The 5 years survival rates were $30 \%$ and $0 \%$, respectively, for chemoradiation and radiation alone. [12 ] Based on the positive results from the RTOG 85-01 trial, the conventional nonsurgical treatment is chemoradiation .[13] Concurrent Paclitaxel,and Cisplatin with radiation are easily infused and well tolerated, regimen with a low evidences of significant esophagitis and a high pathologic response rate consistent with the preclinical data of Paclitaxel and radiation for esophageal carcinoma.[14] Neoadjuvant paclitaxel and cisplatin regimens are well tolerated and also the overall response rates are promising. [15] Esophageal cancer were treated at the Fox Chase Cancer Center on the basis of one of three prospective nonrandomized protocols using concurrent chemotherapy and radiation. Swallowing function was retrospectively assessed in these patients by use of a swallowing-function scoring system. [16-17]

Some studies described toxicities resulting from concurrent chemoradiation in irresectable carcinoma esophagus. One of the eminent German study explicate the toxicities in 22 patients treated with anterior chemoradiation followed by concurrent chemoradiation(chemotherapy with 5FU and cisplatin). The main toxicities were leukocytopenias (23\%) as well as thrombocytopenias (9\%) grade III and IV. [18]

In our institute, many patients refuse radiation at first visit and sometime general condition is too poor to tolerate the adverse effects of concurrent chemoradiation. In such patients, neoadjuvant chemotherapy followed by conventional radiation is delivered.The major purpose of this study was to evaluate the impact of radiation therapy and chemotherapy( applying in different ways in two arms) on swallowing function. The others outcome which have studied were, response for local control, survival and toxicity.

\section{Patients and study design:}

\section{Materials And Method}

This reterospective study was conducted in Acharya tulsi Regional cancer hospital and research institute Bikaner,from September 2011 to December 2013. Patients with upper and middle one-third thoracic esophagus cancer were included in this study and registered. All patients between 25 to 70 years of age, Squamous cell carcinoma histopathologically,clinical stage (AJCC) $\mathrm{T}_{1-3} \quad \mathrm{~N}{ }_{0-1} \mathrm{Mo}$,ECOG Performance score $(0 / 1 / 2)$, without any prior treatment for carcinoma, were randomly distributed among the study arm (25 patients) and control arm(25 patients). Detail History of swallowing function was recorded before, during and after completion of treatment (initial rate of improvement, degree of improvement and long term swallowing function). The scoring system for dysphagia shown in Table 1 and is a modification of the system proposed by O’Rourke et al."

Study arm received concurrent chemotherapy and radiotherapy. Inj.cisplatin $80 \mathrm{mg} / \mathrm{m}^{2}$ in divided doses in day 1 and day 2, repeated after 21 days with Inj.paclitaxel $75 \mathrm{mg} / \mathrm{m} 2$ weekly during external beam radiotherapy (EBRT).(HDR- ICBT ) was delivered 10 to 14 days after the end of EBRT

Control arm received neoadjuvant chemotherapy; Inj-csplatin $80 \mathrm{mg} / \mathrm{m}^{2}$ divided in day 1 and day 2, Inj Paclitaxel $175 \mathrm{mg} / \mathrm{m}^{2}$ on day 1 . Total 2 courses were given at interval of 21 days. External beam radiotherapy (EBRT) was delivered 10 to 14 days after last chemotherapy. High dose rate Intra-cavitory Brachytherapy (HDR- ICBT) was given after 10 to 14 days at the end of EBRT.

Both study and control arms were treated with conventional EBRT 2 Gray per fraction, 5 fraction per week, total 50 Grays ( 25 fraction) in 5 weeks. Anterior-posterior portals were used with $5 \mathrm{~cm}$ safe margin cranio-caudally from clinically visible tumor and $2-3 \mathrm{~cm}$ lateral margins from esophagus (as per pretreatment esophagogram and CT Scan/nodal status). HDR-ICBT was given in 2 fraction each 5 Grays at $0.5 \mathrm{~cm}$. for surface/ $1.2 \mathrm{~cm}$ and $1.1 \mathrm{~cm}$ depth from centre (for $1.4 \mathrm{~cm}$ and $1.2 \mathrm{~cm}$ applicator respectively), 2 days apart, with $3 \mathrm{~cm}$ safe margin cranio-caudily as per pretreatment esophagogram.

Data collection: Patients 'data were collected from institute computerised system. Recorded principle elements were clinical history, age, body surface area (BSA),Body Mass Index(BMI), histopathology of tumor ,endoscopic scrutinies, tumor dimensions ( with CT scan),ECOG performance score .

Toxicity throughout chemoradiation was scored according to Common Terminology Criteria for Adverse Events version 3.0. Also dose modifications or discontinuation of chemo- and radiotherapy were recorded.

\section{Laboratory tests:-}

Complete Blood Count (CBC), Renal Function Test (RTT), Liver function tests (LFT), blood sugar were advised on each course of chemotherapy and on each follow up after treatment. (Total four follows up in 6 months interval of six weeks).

Chest x-ray PA, sonography of whole abdomen, Ba- swallow, were advised prior to starting any protocol and on each follow up.(Total four follows up in 6 months each of interval of six weeks). Upper GIT Endoscopy \& biopsy and Contrast Enhanced Computed Tomography (CECT) of neck, thorax \& upper 
abdomen were advised at baseline,than after 3 months and 6 months of completion of treatment and on further follow up (if required). Ryle's tube feeding, blood transfusion, IV-fluids, antibiotics and other symptomatic/supportive treatment were advised if required.

\section{Statistical analyses}

Descriptive statistics of categorical variables are reported as total numbers and percentages. To compare toxicity between the two groups the chi square test was performed.

\section{Treatment response evaluation and follow up}

Subjective response evaluation (dysphagia) was done weekly during treatment and after completion of treatment till end of 6 month (Total four follows up at interval of six week). Objective response (Acute hematological toxicity, Renal and Liver toxicity, Acute Radiation Toxicity for Lung and esophagus) was done on each course of chemotherapy, at 3 month of the completion of treatment and on further follow ups. The response assessment criteria was based on randomized trial of Japan Clinical Oncology Group (JCOG9906) criteria.For the primary esophageal tumor, Complete Response (CR )was defined as no evidence of residual or recurrent tumor on endoscopy, as verified histologically; all other responses will be defined as Non Complete Response ( non-CR). For lymph nodes, CR was defined as a reduction in lymph-node size from $>1$ to $<1 \mathrm{~cm}$; all other responses will be defined as non-CR. [19]

\section{Swallowing function before treatment:}

\section{Result}

Initial swallowing function (baseline) of all 50 patients of study arm and contrl arm shown in table 1.In both arms there were no asymptomatic patients, all patients having some degree of dysphagia. Before therapy was initiated ,8 patients received intravenous hyperalimentation, 3 patients had feeding tube in place, 2 patients had gastrostomies.

\section{Initial improvement in swallowing function:}

In study arm $22(88 \%)$ of the 25 patients experienced some degree of improvement in swallowing function with time, from time of initial treatment. At the end of 2 weeks 12 patients (48\%) and at the end of treatment (6weeks) 21 patients $(84 \%)$ showed improvement in dysphagia. The range of time for patients improvement was 1-20 weeks. In control arm $16(64 \%)$ of the 25 patients experienced some degree of improvement in dysphagia. At the end of 3 weeks and 6 weeks 8 patients (32\%) and 10 patients $(40 \%)$ respectively showed improvement in dysphagia. At the end of 12 weeks i.e. completion of treatment $16(64 \%)$ patients showed improment in dysphagia.

Table 1.Initial swallowing function of two study groups

\begin{tabular}{|l|l|l|}
\hline Swallowing score* & $\begin{array}{l}\text { Study arm } \\
\text { no. of patients(\%) }\end{array}$ & $\begin{array}{l}\text { Control arm } \\
\text { no. of patients(\%) }\end{array}$ \\
\hline 1.Asymptomatic & 0 & 0 \\
\hline 2.Difficult swallowing of solid & $12(48 \%)$ & $8(32 \%)$ \\
\hline 3. Difficult swallowing of semisolid & $7(28 \%)$ & $10(40 \%)$ \\
\hline 4.Drink liquid only & $5(20 \%)$ & $4(16 \%)$ \\
\hline 5.Abslute dysphagia & $1(4 \%)$ & $3(12 \%)$ \\
\hline
\end{tabular}

Modified $\mathrm{O} `$ Rourke et al $*{ }^{12}$

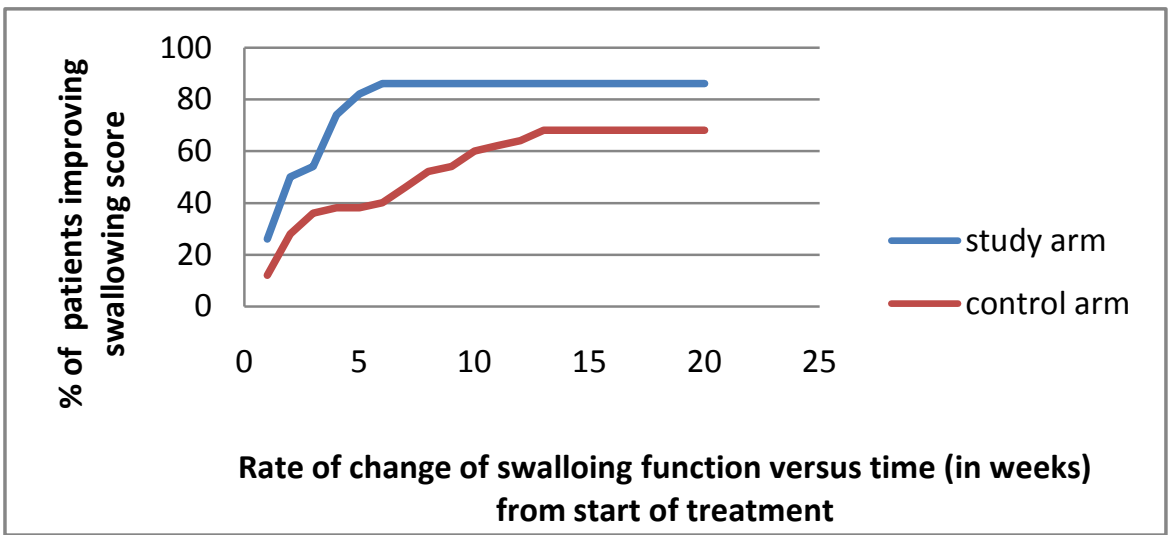

Table 2 - Acute Hematological toxicities

\begin{tabular}{|l|l|l|l|l|l|}
\hline Level of toxicity & $\mathbf{0}$ & $\mathbf{1}$ & $\mathbf{2}$ & $\mathbf{3}$ & $\mathbf{4}$ \\
\hline
\end{tabular}


Superiority Of Concurrent Chemo Radiation Over Sequential Chemo Radiotherapy For....

\begin{tabular}{|c|c|c|c|c|c|c|c|}
\hline \multirow{6}{*}{ Hb } & \multirow[t]{2}{*}{ Study } & $\mathrm{n}=25$ & 1 & 12 & 10 & 2 & 0 \\
\hline & & $\%$ & 4 & 48 & 40 & 8 & - \\
\hline & \multirow[t]{2}{*}{ Control } & $\mathrm{n}=25$ & 1 & 18 & 4 & 2 & 0 \\
\hline & & $\%$ & 4 & 72 & 16 & 8 & - \\
\hline & \multicolumn{2}{|c|}{$\chi^{2}$} & \multicolumn{5}{|c|}{1.634} \\
\hline & \multicolumn{2}{|c|}{$\mathrm{P}$} & \multicolumn{5}{|c|}{0.65} \\
\hline \multirow{6}{*}{ TLC } & \multirow[t]{2}{*}{ Study } & $\mathrm{n}=25$ & 4 & 11 & 8 & 2 & 0 \\
\hline & & $\%$ & 16 & 44 & 32 & 8 & - \\
\hline & \multirow[t]{2}{*}{ Control } & $n=25$ & 11 & 13 & 0 & 1 & 0 \\
\hline & & $\%$ & 44 & 52 & - & 4 & - \\
\hline & \multicolumn{2}{|c|}{$\overline{\chi^{2}}$} & \multicolumn{5}{|c|}{8.576} \\
\hline & \multicolumn{2}{|c|}{$\mathrm{p}$} & \multicolumn{5}{|c|}{0.013} \\
\hline \multirow{6}{*}{ ANC } & \multirow[t]{2}{*}{ Study } & $n=25$ & 7 & 11 & 5 & 2 & 0 \\
\hline & & $\%$ & 28 & 44 & 20 & 8 & - \\
\hline & \multirow[t]{2}{*}{ Control } & $n=25$ & 12 & 13 & 0 & 0 & 0 \\
\hline & & $\%$ & 48 & 52 & - & - & - \\
\hline & \multicolumn{2}{|c|}{$\chi^{2}$} & \multicolumn{5}{|c|}{4.569} \\
\hline & \multicolumn{2}{|c|}{$\mathrm{p}$} & \multicolumn{5}{|c|}{0.014} \\
\hline \multirow{6}{*}{ Platelets } & \multirow[t]{2}{*}{ Study } & $\mathrm{n}=25$ & 17 & 8 & 0 & 0 & 0 \\
\hline & & $\%$ & 68 & 32 & - & - & - \\
\hline & \multirow[t]{2}{*}{ Control } & $\mathrm{n}=25$ & 22 & 3 & 0 & 0 & 0 \\
\hline & & $\%$ & 88 & 12 & - & - & - \\
\hline & \multicolumn{2}{|c|}{$\overline{\chi^{2}}$} & \multicolumn{5}{|c|}{2.914} \\
\hline & & & & & & & \\
\hline
\end{tabular}

Table 2 shows grades of acute hematological toxicities including Hb, TLC, ANC and Platelets counts, that observed according to the RTOG Criteria. Grades of all toxicities that are mentioned here are the highest hematological toxicity grade per patient that were observed in whole study. Majority of patients had grade 0,1 , and 2 toxicities in both arms and these were well tolerated $(\mathrm{p}=0.65$, and $\mathrm{p}=0.087$ respectively for $\mathrm{Hb}$ and Platelets counts). There was statistical significant difference in toxicities of TLC and ANC between both arms. Grade 2 and 3 toxicities were $40 \%$ and $4 \%$ for TLC and were $28 \%$ and $0 \%$ for ANC in study and control arm respectively ( $\mathrm{p}=0.013, \mathrm{p}=.014)$. This may be due to concurrent use of chemotherapy with radiation in study arm that also showed synergism for toxicity of TLC and ANC.

Table 3- Response of Primary Esophageal Tumour and mediastinal tumor (6 Months)

\begin{tabular}{|c|c|c|c|c|c|c|c|c|}
\hline \multirow[t]{3}{*}{ Response } & \multicolumn{4}{|c|}{ Study Arm } & \multicolumn{4}{|c|}{ Control Arm } \\
\hline & \multicolumn{2}{|c|}{ Male } & \multicolumn{2}{|c|}{ Female } & \multicolumn{2}{|c|}{ Male } & \multicolumn{2}{|c|}{ Female } \\
\hline & $n=9$ & $\%$ & $n=16$ & $\%$ & $\mathrm{n}=12$ & $\%$ & $n=13$ & $\%$ \\
\hline $\begin{array}{c}\text { Primary }=\mathrm{CR} \\
\text { Lymph node }=\mathrm{CR}\end{array}$ & 7 & 28 & 13 & 52 & 10 & 40 & 7 & 28 \\
\hline $\begin{array}{c}\text { Primary }=\mathrm{CR} \\
\text { Lymph node=Non-CR }\end{array}$ & 0 & 0 & 0 & 0 & 0 & 0 & 0 & 0 \\
\hline $\begin{array}{l}\text { Primary }=\text { Non }-C R \\
\text { Lymph node }=C R\end{array}$ & 0 & 0 & 1 & 4 & 0 & 0 & 1 & 4 \\
\hline $\begin{array}{c}\text { Primary=Non-CR } \\
\text { Lymph node=Non-CR }\end{array}$ & 2 & 8 & 2 & 8 & 2 & 8 & 5 & 20 \\
\hline
\end{tabular}

Table 3 shows CR and Non-CR for primary esophageal tumor as per response criteria of JCOG 9906 trial. ${ }^{19}$ Assessment of response in both primary tumor and mediastinal lymph nodes was done after 6 months of completion of the treatment with CT scan. CR was observed in 20 patients (80\% of all) of study arm and in 17 patients $(68 \%)$ of control arm $(\mathrm{p}>0.05)$. In study arm $28 \%$ male and $52 \%$ female patients, while in control arm $40 \%$ male and $28 \%$ female patients presented with CR in primary tumor and mediastinal lymph node.

\section{Discussion}

This study analysize that nearly $90 \%$ patients of carcinoma esophagus showed improvement from dysphagia with concurrent chemoradiation,time to initial improvement is not related to stage of tumor. This regimen has not only provided rapid early relief from dysphagia but also showed long term near normal swallowing function. In our study, incidence of overall maximum toxicities among the study and control arm were as follows; grade I $12 \%$ v/s $32 \%$ toxicity, grade II $56 \%$ vs $48 \%$ toxicity, grade III $32 \%$ vs $20 \%$ toxicity, grade IV and $0 \%$ vs $0 \%$ toxicity respectively .Study arm had higher incidence of grade 2 and 3 overall toxicity, while grade I overall toxicity was dominant in control arm. We also observed higher incidence of radiation induced toxicities in study arm as compare to control arm. Grade I pneumonitis was in $32 \%$ and $24 \%$ respectively,grade II pneumonitis was $8 \%$ in each arm $(\mathrm{p}=0.838)$, while grade I esophagitis was $40 \%$ and $32 \%$, grade II esophagitis $40 \%$ and $48 \%$,GradeIIIesophagitis was $20 \%$ and $8 \%$ in study arm and control arm 
respectively $(\mathrm{p}=0.416)$. None of patient in our study presented with grade III pneumonitis and grade IV esophagitis. Renal and liver toxicities were mainly of grade 0 or I and well tolerated in patients of both arms.

Comparison of overall toxicities was also done with age group, performance status, sex and grade of dysphagia at presentation. We observed that overall grade $>3$ toxicities were less in patients of control arm as compared to study arm i.e. $32 \%$ vs $20 \%$ respectively ( $\mathrm{p}=0.993$ ). Toxicities were almost equal in both type of sex groups and well tolerated. Patients that presented with grade $>I V$ dysphagia had more grade 2 and 3 toxicities in study arm as well as control arm $(87 \% \mathrm{v} / \mathrm{s} 100 \% \mathrm{p}=.992)$. These toxicities may be due to nil or poor oral intake of liquid and calories that further enhanced by concurrent chemoradiation in such patients.

Evaluation of treatment response was done after 6 months of completion of treatment in terms of local control (complete response at primary site) and regional control (complete response at lymph node site) as per assessment criteria of JCOG 9906 trial. [20] Results in term of CR was superior in study arm but there was no statistically significant difference between both arms $(\mathrm{p}=0.841)$. At primary site, complete response $(\mathrm{CR})$ observed in $80 \%$ patients of study arm while it was in $68 \%$ patients of control arm $(\mathrm{p}=0.529)$. At mediastinal lymph node site, CR was observed in 81.82 patients of study arm while it was in $69.57 \%$ patients of control arm $(\mathrm{p}=0.339)$ INT 0123 trial approximately matches with our study arm. Local failure and regional failure observed in $20 \%$ and $18.18 \%$ patients respectively in our study arm while it were $33 \%$ and $23 \%$ respectively in INT 0123 trial. [21] Herskovic et al.[22] randomized 240 patients into study and control arm with similar treatment regime of INT 0123 trial or RTOG 85-06 trial. Results of our study also match with this trial. In our study, local failure and regional failure was $20 \%$ and $18.18 \%$, while $22 \%$ and $24 \%$ in this trial respectively.

Ilson et al [23] conducted a trial on neoadjuvant chemotherapy followed by radiation. All patients received two course of chemotherapy (Cisplatin \& $5 \mathrm{FU}$ ). Treatment was well tolerated all patient and none of the patient present with grade III toxicity. They summarized that neoadjuvant chemotherapy followed by radiation is a better treatment regime for elderly people and such patients whose general condition can't tolerate higher grade toxicities due to higher grade of dysphagia and poor nutrition. Observations of toxicity of our study closely match with above study.

In short, concurrent chemoradiation (treatment of study arm) was superior in term of , better alleviation of dysphagia ,local and regional control along with short duration of treatment as compare to control arm. On other hand, neoadjuvant chemotherapy followed by radiotherapy (treatment of control arm) was better in term of lower incidence of grade $>3$ acute toxicities especially for elderly age group or patient with grade $>4$ dysphagia. Statistical evaluation of our results with the standard studies indicates that observed values correlate closely with standard results. May be larger studies in terms of number of patients and follow up tenure will yield conclusive results

\section{Summary And Conclusion:}

Long ago, it was not clear whether radiation alone or concurrent chemoradiation is better treatment of esophageal cancer. But now a days if we feel that patient can tolerate the combined modality, then it wise to use concurrent chemoradiotherapy .Role of chemotherapy in this setting as a radiosensitizer, local control and, in some cases, survival, by mutilating the radioresistant territories . In our study, palliation of dysphagia was $88 \%$ vs $64 \%$ in study arm (concurrent chemoradiation) and control arm (sequential chemoradiation) respectively $(\mathrm{p}=0.333)$.

No doubt, incidence of grade $>2$ toxicities is higher in concurrent chemoradiation as compared to sequential chemoradiation. We observed grade 3 toxicity $32 \%$ vs $20 \%$ in study and control arm respectively $(\mathrm{p}=0.804)$. This is very important in such patients who are elderly or having severe dysphagia. In our study, elderly patient had grade $>2$ toxicity in study arm as compared to control arm $(\mathrm{p}=0.993)$. Similarly, patients with grade $>4$ dysphagia had higher incidence of severe toxicities in study arm as compared to control arm. Such patients have deficiency of nutrition \& energy and shows very poor tolerance for concurrent chemoradiation. So this group could be treated with sequential chemoradiation.

\section{Bibliography}

[1]. Coorly DA, Buffer PA, Oesophageal and gastic cardia adenocarcinoma; analysis of regional variation using the cancer incidence database.Int J Epidemol 2001; 30:1414-1425.

[2]. Brown LM,Devesa SS.Epidemiogic trends in esophageal and gastric cancer in the United States. Surg Oncol Clin Nm 2002;11(2):235.

[3]. American Joint Commission on Cancer 6th ed. AJCC Cancer Staging Manual. 6th ed. New York: Springer Verlag, 2002.

[4]. Rosenberg J, Lichter A, Leichman L. Cancer of the esophagus, 3rd ed. Philadelphia: J.B. Lippincott, 1989.

[5]. Torre LA, Bray F, Siegel RL, Ferlay J, Lortet-Tieulent J, Jemal A. Global cancer statistics, 2012. CA Cancer J Clin. 2015;65:87108.

[6]. Daly JM, Karnell LH, Menck HR. National cancer database report on esophageal carcinoma. Cancer 1996;78:1820.

[7]. 90. Mariette C, Balon JM, Piessen G, et al. Pattern of recurrence following complete resection of esophageal carcinoma and factors predictive of recurrent disease. Cancer 2003;97(7):1616.

[8]. Dai Y, Li C, Xie Y, et al. Interventions for dysphagia in oesophageal cancer. Cochrane Database Syst Rev. 2014;10:CD005048. 
[9]. Gebski V, Burmeister B, Smithers BM, et al: Survival benefits from neoadjuvant chemoradiotherapy or chemotherapy in oesophageal carcinoma: a meta-analysis. Lancet Oncol. 2007, 8: 226-234. 10.1016/S1470-2045(07)70039-6.

[10]. van Hagen P, Hulshof MC, van Lanschot JJ, et al: Preoperative chemoradiotherapy for esophageal or junctional cancer. N Engl J Med. 2012, 366: 2074-2084. 10.1056/NEJMoa1112088.

[11]. Cooper JS, Guo MD, Herskovic A, et al: Chemoradiotherapy of locally advanced esophageal cancer: long-term follow-up of a prospective randomized trial (RTOG 85-01). Radiation Therapy Oncology Group. JAMA. 1999, 281: 1623-1627

[12]. Al-Sarraf M, Martz K, Herskovic A, et al. Progress report of combined chemoradiotherapy versus radiotherapy alone in patients with esophageal cancer: an Intergroup study. J Clin Oncol 1997;15:277â€“284.

[13]. Herskovic A, Martz K, Al-Sarraf M, et al. Combined chemotherapy radiotherapy compared with radiotherapy alone in patients with cancer of the esophagus. N Engl J Med 1992;326(24):1593.

[14]. The Brown University Oncology Group, Providence, Rhode Island, USA.

[15]. Ann Oncol (2003) 14 (8): 1253-1257. doi: 10.1093/annonc/mdg328.

[16]. Lawrence R. Coia M.D.1,*, Edward M. Soffen M.D.2, Timothy E. Schultheiss Ph.D.1, Eric E. Martin B.S.E.1 andGerald E. Hanks M.D.1Swallowing function in patients with esophageal cancer treated with concurrent radiation and chemotherapyVersion of Record online: 28 JUN 2006DOI: 10.1002/1097-0142(19930115)71:2-281::AID-CNCR2820710202-3.0.CO;2-0.

[17]. I. C. O'Rourke,K. Tiver ,C. Bull ,V. Gebski , Langlands .Swallowing performance after radiation therapy for carcinoma of the esophagus, 10.1002/10970142(19880515)61:10-2022::AIDCNCR2820611016-3.0.C.

[18]. Stuschke M, Stahl M, Wilke H, et al: Induction chemotherapy followed by concurrent chemotherapy and high-dose radiotherapy for locally advanced squamous cell carcinoma of the upper-thoracic and midthoracic esophagus. Am J Clin Oncol. 2000, 23: 233-238. 10.1097/00000421-200006000-00004.

[19]. Ken Kato,Takako Eguchi Nakajima,Yoshinori Ito,Chikatoshi Katada

[20]. Hiromichi Ishiyama,Shin-ya Tokunaga,Masahiro Tanaka,Shuichi HironakaTakayuki Hashimoto,Takashi Ura. Phase II Study of Concurrent Chemoradiotherapy at the Dose of 50.4 Gy with Elective Nodal Irradiation for Stage II-III Esophageal Carcinoma.Jpn J Clin Oncol (2013) 43 (6): 608-615.

[21]. Safran H, DiPetrillo T, Nadeem A, et al. Neoadjuvant herceptin paclitaxel,cisplatin, and radiation for adenocarcinoma of esophagus: a phase I study. Proc Am Soc Clin Oncol 2002;21:141a.

[22]. Herskovic A, Martz K, Al-Sarraf M, et al. Combined chemotherapy and radiotherapy compared with radiotherapy alone in patients with cancer of the esophagus. N Engl J Med 1992;326(24):1593.

[23]. Gaspar LE, Qian C, Kocha WI, et al. A phase I/II study of external beam radiation, brachytherapy and concurrent chemotherapy in localized cancer of the esophagus (RTOG 92-07): preliminary toxicity report. Int J Radiat Oncol Biol Phys 1997;37:593. 